\title{
Multimodel magnetic resonance imaging of mass-forming autoimmune pancreatitis: differential diagnosis with pancreatic ductal adenocarcinoma
}

\author{
Huihui Jia', Jialin Li ${ }^{2}$, Wenjun Huang ${ }^{1}$ and Guangwu Lin ${ }^{1 *}$
}

\begin{abstract}
Purpose: To assess the value of the multimodel magnetic resonance imaging (MRI), including unenhanced images, dynamic contrast-enhanced MRI (DCE-MRI), MR-cholangiopancreatography (MRCP), and diffusion-weighted imaging (DWI), in differentiation of mass-forming autoimmune pancreatitis (AIP) from pancreatic ductal adenocarcinoma (PDAC).
\end{abstract}

Methods: Twelve patients with mass-forming AIP and 30 with PDAC were included. All patients underwent unenhanced MRI, DCE-MRI, DWI, and MRCP. Relevant values including sensitivity and specificity of the imaging features and their diagnostic performance for predicting mass-forming AIP were analyzed.

Results: Several statistically significant MR findings and quantitative indexes differentiating mass-forming AIP from PDAC, including multiplicity, irregularity or conformation, capsule-like rim enhancement, absence of internal cystic or necrotic portion, homogeneous enhancement during pancreatic, venous, and delayed phases, skipped stricture or stricture of MPD, absence of side branch dilation, maximum upstream MPD diameter $<2.4 \mathrm{~mm}$, Contrast $\mathrm{uP}_{\mathrm{P}}>0.739$,

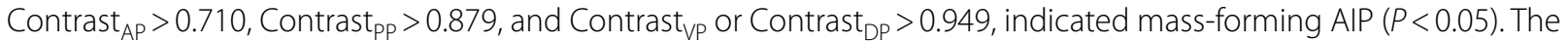
apparent diffusion coefficient (ADC) value was also significantly lower in mass-forming AIP compared to that in PDAC $(P=0.006)$. The cutoff value of ADC for distinguishing mass-forming AIP from PDAC was $1.099 \times 10^{-3} \mathrm{~mm}^{2} / \mathrm{s}$.

Conclusion: Multimodel MRI, including unenhanced MRI, DCE-MRI with DWI and MRCP can provide qualitative and quantitative information about mass-forming AIP characterization. Multimodel MRI are valuable for differentiating mass-forming AIP from PDAC.

Keywords: Magnetic resonance imaging, Autoimmune pancreatitis, Pancreatic ductal adenocarcinoma, Diffusionweighted imaging

\footnotetext{
*Correspondence: lingw01000@163.com

1 Department of Radiology, Huadong Hospital Affiliated to Fudan

University, 221 West Yanan Road, Shanghai 200040, China

Full list of author information is available at the end of the article
}

\section{Background}

Autoimmune pancreatitis (AIP) is a distinct type of chronic fibroinflammatory pancreatitis characterized by abundant lymphoplasmacytic infiltration, interstitial fibrosis, and elevated serum levels of IgG4 $[1,2]$. The etiology of AIP is yet unclear [2-4]. According to the different clinicopathological characteristics, AIP is now divided into type 1 and type 2 subtypes [2,5]. Type 1 is an 
IgG4-related systemic disease that can have extra-pancreatic involvement $[5,6]$. Type 2 is characterized histologically by idiopathic duct-centric pancreatitis [6]. Type 2 is not associated with either serum IgG4 elevation or extrapancreatic involvement [5].

In the event of diffuse involvement, the enlarged pancreas manifests a "sausage-like" appearance, which is different from that of pancreatic ductal adenocarcinoma (PDAC) $[7,8]$. Mass-forming AIP accounts for approximately $28-41 \%$ of all cases of AIP $[9,10]$. Mass-forming AIP sometimes is difficult to differentiate from PDAC as the two diseases have the overlapping clinical and radiological features. AIP responds dramatically to steroid therapy, while PDAC is curable only by surgical resection $[1,11]$. Furthermore, the differentia between the two types of diseases is crucial as treatment and prognosis are different.

In this study, the diagnostic dilemma of differentiating between these two entities is discussed. In addition, we review the characteristics of each condition by multimodel magnetic resonance imaging (MRI), including unenhanced MRI, dynamic contrast-enhanced MRI (DCE-MRI), MR-cholangiopancreatography (MRCP), and diffusion-weighted imaging (DWI) findings, for a better understanding of the two conditions.

\section{Methods \\ Patients}

This retrospective study was approved by the Ethics Committee of Fudan University and all the procedures were conducted in accordance with the Declaration of Helsinki of 1996. From our institution's medical database between January 2015 and February 2020, 37 patients diagnosed with AIP were found in the study based on the diagnostic criteria [12-14]. Twenty-five patients with AIP were excluded: 8 had only undergone computed tomography $(\mathrm{CT})$ scan, 10 with only unenhanced MRI images without dynamic enhancement, and 1 with only dynamic enhancement without unenhanced MRI images, while the remaining 6 patients were diffuse type AIP. Finally, 12 patients with mass-forming AIP, according to the histopathological confirmation at surgery or biopsy $(n=7)$ or by the combination of elevated serum IgG4 level, typical imaging features, and response dramatically well to steroid therapy $(\mathrm{n}=5)$, were included in this study. Additionally, data from 30 PDAC patients with pathologically confirmed between January 2019 and February 2020 were also analysed (Fig. 1).

\section{MRI techniques}

MRI was performed using a $3.0 \mathrm{~T}$ scanner (Magnetom Skyra, Siemens, Erlangen, Germany) with an 8-channel body-phased array coil. T2-weighted

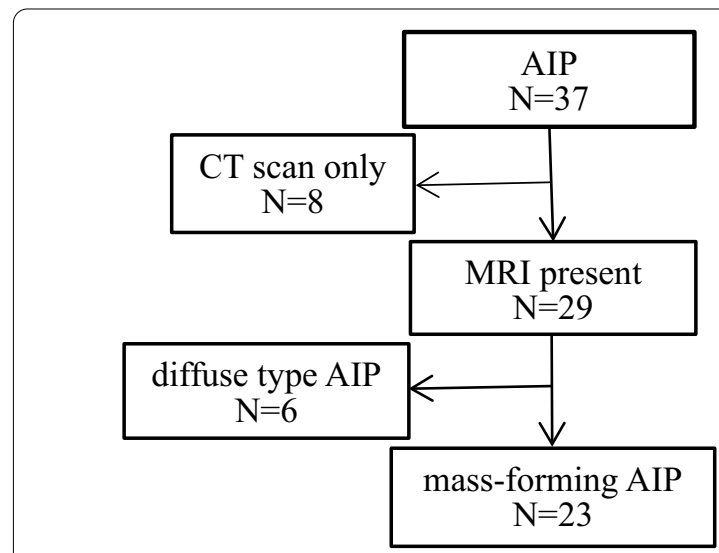

Fig. 1 Flow diagram of study enrollment population

half-Fourier acquisition single-shot turbo spin-echo (HASTE) sequence, navigator-triggered fat-suppressed T2-weighted turbo spin-echo sequence, MRCP images, breath-hold T1-weighted Dixon vibe sequence, DWI and dynamic imaging with a three-dimensional volumetric interpolated breath-hold examination (3D-VIBE) sequence were included (Table 1). 3D-VIBE sequence was obtained before (unenhanced phase) and $20 \mathrm{~s}$ (arterial phase, AP), $35 \mathrm{~s}$ (pancreatic phase, $\mathrm{PP}$ ), $50 \mathrm{~s}$ (portal venous phase, $\mathrm{VP}$ ), and 3-6 min (delayed phase, DP) after intravenous administration of gadopentetate dimeglumine (Magnevist; Bayer Schering Pharma). The contrast agent was injected intravenously as a bolus $(3.0 \mathrm{~mL} / \mathrm{s})$ at a dose of $0.1 \mathrm{mmol} / \mathrm{kg}$ followed by flushing with $20 \mathrm{~mL}$ saline using a power injector. The apparent diffusion coefficient (ADC) value was calculated with b values 50 and $800 \mathrm{~s} / \mathrm{mm}^{2}$, respectively. The average interval between MRI and surgery was 10 days (range, $1-30$ days).

\section{Image analysis}

The MR images were reviewed independently by two radiologists (GWL and HHJ with 15 and 7 years of experience in abdominal imaging, respectively) who were blinded to the results aside from the diagnosis including AIP and PDAC. In case of disagreements, the discussion was continued until a final consensus was reached.

Items analyzed for each mass included the number of masses, location (uncinate, head or neck, body or tail), margin (indistinct, distinct), contour (focal and diffuse enlargement, focal protrusion, or no bulging), shape (oval or round, irregular, or geographic), capsule-like rim, internal cystic or necrotic component, upstream pancreatic atrophy, peripancreatic infiltration, vascular invasion, lymphadenopathy, T2WI signal intensity (SI) (hypointense, isointense, and hyperintense), T1WI SI, 
Table $1 \mathrm{MRI}$ sequences and parameters

\begin{tabular}{|c|c|c|c|c|c|c|c|}
\hline & \multicolumn{2}{|l|}{$\mathrm{T} 2 \mathrm{WI}$} & \multirow[t]{2}{*}{ T1WI } & \multirow{2}{*}{$\begin{array}{l}\text { Thick slab single } \\
\text { slice MRCP }\end{array}$} & \multirow{2}{*}{$\begin{array}{l}\text { Thin slab } \\
\text { multislice MRCP }\end{array}$} & \multirow[t]{2}{*}{ 3D VIBE } & \multirow[t]{2}{*}{ DWI } \\
\hline & HASTE & RT-TSE & & & & & \\
\hline TR/TE (ms) & $1600 / 95$ & $3000 / 79$ & $4 / 1.29$ & $4500 / 979$ & $1150 / 252$ & $3.55 / 1.27$ & $4700 / 66$ \\
\hline$F A\left({ }^{\circ}\right)$ & $150^{\circ}$ & $120^{\circ}$ & $9^{\circ}$ & $180^{\circ}$ & $121^{\circ}$ & $13^{\circ}$ & - \\
\hline Section thickness (mm) & 5 & 5 & 3 & 45 & 4 & 2.5 & 6 \\
\hline Matrix & $320 \times 256$ & $320 \times 256$ & $320 \times 256$ & $512 \times 320$ & $512 \times 409$ & $320 \times 256$ & $192 \times 153$ \\
\hline $\mathrm{FOV}(\mathrm{cm})$ & $32-38$ & $32-38$ & $32-38$ & 30 & 32 & $32-38$ & $32-38$ \\
\hline Acquisition time (s) & 60 & 150 & 16 & 5 & 20 & 18 & 198 \\
\hline
\end{tabular}

T2WI T2-weighted images, T1WIT1-weighted images, MRCP magnetic resonance cholangiopancreatography, 3D VIBE three-dimensional volumetric interpolated breath-hold examination, DWI diffusion-weighted imaging, HASTE half-Fourier acquisition single-shot turbo spin-echo, RT-TSE respiratory-triggered turbo spin-echo, $T R$ repetition time, $T E$ echo time, FA flip angle, FOV field of view

DWI SI, and homogeneity of enhancement or SI in AP, PP, VP, and DP (homogeneous, heterogeneous).

In addition, the MRCP findings were analysed to assess the potential existence of abnormalities of the common bile duct $(\mathrm{CBD})$ and pancreatic ducts. The following characteristics were analyzed: complete obstruction of $\mathrm{CBD} /$ main pancreatic duct (MPD), the stricture or skipped stricture of CBD/MPD, the sidebranch dilation, and the presence of penetrating duct sign. The penetrating duct sign indicates the MPD penetrating the mass. In the case of multiple lesions in one patient, an image analysis of each lesion characteristic was performed for that patient.

Quantitative analysis of the mass enhancement contrast in AIP and PDAC was performed by another reviewer (WJH, 6 years of experience) who did not participate in the qualitative image analysis. A region-ofinterest (ROI) was drawn to assess SI of the pancreatic mass and non-mass adjacent pancreatic parenchyma (NAP) on DCE-MRI. The ROI was lesion-size-dependent for the pancreatic mass. In NAP, the ROI was placed downstream to the mass to exclude any potential obstructive pancreatitis-related SI change. Three measurements were performed for the same image in each phase. The average SI values were calculated to obtain the mass enhancement contrast at each phase: Contrast $=\mathrm{SI}_{\text {mass }} / \mathrm{SI}_{\mathrm{NAP}}$.

Furthermore, lesion diameter and ADC were measured for quantitative analysis. An ROI was drawn in areas of the pancreatic mass and the NAP for the measurement of ADC, devoid of cystic or necrotic portions. Subsequently, the normalized ADC was calculated: $\mathrm{ADC}_{\text {mass }} / \mathrm{ADC}_{\mathrm{NAP}}$, where $A D C_{\text {mass }}$ is the $\mathrm{ADC}$ of the pancreatic mass and $\mathrm{ADC}_{\mathrm{NAP}}$ is the $\mathrm{ADC}$ of the NAP. The ADC maps were available for 16 masses of massforming AIP and 30 masses of PDAC. Also, the maximum upstream MPD diameter was also measured in all patients.

\section{Statistical analysis}

Pearson's chi-square test and Fisher's exact test were performed to compare categorical variables between AIP and PDAC groups. Student's t-test was performed for continuous variables. Interobserver variability regarding the qualitative parameters was assessed by using Kappa test. The strength of agreement was assessed as follows: $\mathrm{k}$ values $<0.20$ indicated poor agreement; $\mathrm{k}$ values of $0.21-0.40$ fair agreement; $k$ values of $0.41-0.60$ moderate agreement; $\mathrm{k}$ values of $0.61-0.80$ good agreement; $\mathrm{k}$ values of 0.81-1.00 excellent agreement. Student's t-test was used to compare the mass enhancement contrast at each phase of DCE-MRI between the two groups. Receiver operating characteristic (ROC) was employed to analyze the diagnostic performance of the lesion enhancement contrast at each phase in distinguishing AIP from PDAC. The optimal cutoff point of the mass enhancement contrast was determined to maximize both the sensitivity and specificity (i.e., Youden index). The sensitivity and specificity of each imaging finding was calculated. MedCalc version 19.1 software (MedCalc Software, Mariakerke, Belgium) and SPSS version 22.0 (IBM SPSS Inc., Chicago, IL, USA) were used for statistical analysis. $P<0.05$ indicated statistical significance.

\section{Results \\ Qualitative analysis of MRI features}

For qualitative analysis, MRI characteristics are summarized in Table 2. Multiple pancreatic masses were significantly more prevalent in AIP (3 patients with two lesions and 1 with three lesions) than in PDAC without multiple lesions $(P=0.004)$ (Figs. 2, 3). Mass-forming AIP $(9 / 17,52.9 \%)$ constantly exhibited an irregular or geographical morphology, while PDAC manifested either an oval or round shape $(27 / 30,90.0 \%)(P=0.004)$. The sign of capsule-like rim was presented exclusively in AIP patients $(6 / 17,35.3 \%)$, while internal cyst or necrotic 
Table 2 MRI findings

\begin{tabular}{|c|c|c|c|c|c|}
\hline & $\begin{array}{l}\text { AIP }(N=17 \text { masses in } 12 \\
\text { patients })\end{array}$ & $k$ & $\operatorname{PDAC}(N=30)$ & $k$ & $P$ value \\
\hline Number of masses & & 0.824 & & 1 & 0.004 \\
\hline 1 & $8 / 12$ & & $30 / 30$ & & \\
\hline 2 & $3 / 12$ & & $0 / 30$ & & \\
\hline 3 & $1 / 12$ & & $0 / 30$ & & \\
\hline Location & & 0.904 & & 0.820 & 0.343 \\
\hline Uncinate & $3 / 17$ & & $2 / 30$ & & \\
\hline Head or neck & $6 / 17$ & & $16 / 30$ & & \\
\hline Body or tail & $8 / 17$ & & $12 / 30$ & & \\
\hline Margin & & 0.876 & & 1 & 0.214 \\
\hline Indistinct & $7 / 17$ & & $18 / 30$ & & \\
\hline Distinct & $10 / 17$ & & $12 / 30$ & & \\
\hline Contour & & 1 & & 0.776 & 0.173 \\
\hline Focal and diffuse enlargement & $3 / 17$ & & $4 / 30$ & & \\
\hline Focal protrusion & $9 / 17$ & & $23 / 30$ & & \\
\hline No bulging & $5 / 17$ & & $3 / 30$ & & \\
\hline Shape & & 0.881 & & 0.839 & 0.004 \\
\hline Oval or round & $8 / 17$ & & $27 / 30$ & & \\
\hline Irregular or geographical & $9 / 17$ & & $3 / 30$ & & \\
\hline Capsule-like rim & $6 / 17$ & 0.866 & $0 / 30$ & 1 & 0.001 \\
\hline Internal cystic or necrotic portion & $0 / 17$ & 1 & $10 / 30$ & 0.923 & 0.008 \\
\hline Upstream pancreatic atrophy & $2 / 12$ & 1 & $10 / 30$ & 0.700 & 0.453 \\
\hline Peripancreatic infiltration & $1 / 12$ & 1 & $12 / 30$ & 0.789 & 0.067 \\
\hline Vascular invasion & $2 / 12$ & 0.625 & $10 / 30$ & 0.923 & 0.453 \\
\hline Lymphadenopathy & $2 / 12$ & 1 & $3 / 30$ & 1 & 0.613 \\
\hline T2WI signal intensity & & 0.767 & & 1 & 0.128 \\
\hline Isointense & $3 / 17$ & & $1 / 30$ & & \\
\hline Hyperintense & $14 / 17$ & & $29 / 30$ & & \\
\hline T1WI signal intensity & & 0.767 & & 1 & 1.000 \\
\hline Hypointense & $16 / 17$ & & $29 / 30$ & & \\
\hline Isointense & $1 / 17$ & & $1 / 30$ & & \\
\hline DWI signal intensity & & 0.821 & & 0.902 & 1.000 \\
\hline Hyperintense & $14 / 17$ & & $24 / 30$ & & \\
\hline Isointense & $3 / 17$ & & $6 / 30$ & & \\
\hline \multicolumn{6}{|c|}{ Signal intensity and enhancement of mass } \\
\hline \multicolumn{6}{|c|}{ The arterial phase } \\
\hline Hypointense & $15 / 17$ & 1 & $29 / 30$ & 1 & 0.544 \\
\hline Isointense & $2 / 17$ & & $1 / 30$ & & \\
\hline Hyperintense & $0 / 17$ & & $0 / 30$ & & \\
\hline Homogeneous & $4 / 17$ & 0.821 & $2 / 30$ & 1 & 0.170 \\
\hline Heterogeneous & $13 / 17$ & & $28 / 30$ & & \\
\hline \multicolumn{6}{|l|}{ The pancreatic phase } \\
\hline Hypointense & $9 / 17$ & 0.901 & $25 / 30$ & 0.621 & 0.017 \\
\hline Isointense & $3 / 17$ & & $4 / 30$ & & \\
\hline Hyperintense & $5 / 17$ & & $1 / 30$ & & \\
\hline Homogeneous & $8 / 17$ & 0.764 & $2 / 30$ & 1 & 0.002 \\
\hline Heterogeneous & $9 / 17$ & & $28 / 30$ & & \\
\hline \multicolumn{6}{|l|}{ The venous phase } \\
\hline Hypointense & $2 / 17$ & 1 & $23 / 30$ & 0.918 & $<0.001$ \\
\hline Isointense & $2 / 17$ & & $4 / 30$ & & \\
\hline
\end{tabular}


Table 2 (continued)

\begin{tabular}{|c|c|c|c|c|c|}
\hline & $\begin{array}{l}\text { AIP }(N=17 \text { masses in } 12 \\
\text { patients) }\end{array}$ & $k$ & $\operatorname{PDAC}(N=30)$ & $k$ & $P$ value \\
\hline Hyperintense & $13 / 17$ & & $3 / 30$ & & \\
\hline Homogeneous & $12 / 17$ & 0.866 & $2 / 30$ & 1 & $<0.001$ \\
\hline Heterogeneous & $5 / 17$ & & $28 / 30$ & & \\
\hline \multicolumn{6}{|l|}{ The delayed phase } \\
\hline Hypointense & $1 / 17$ & 1 & $15 / 30$ & 0.725 & 0.001 \\
\hline Isointense & $1 / 17$ & & $4 / 30$ & & \\
\hline Hyperintense & $15 / 17$ & & $11 / 30$ & & \\
\hline Homogeneous & $13 / 17$ & 0.850 & $2 / 30$ & 1 & $<0.001$ \\
\hline Heterogeneous & $4 / 17$ & & $28 / 30$ & & \\
\hline
\end{tabular}

Numbers represent the number of patients

portion $(P=0.008)$ was commonly observed in the PDAC patients.

According to dynamic imaging, several AIP and PDAC lesions showed hypointense and heterogeneous enhancement during the AP, albeit no significant difference was detected between AIP and PDAC groups $(P>0.05)$. However, PDAC more frequently presented a heterogeneous enhancement (Figs. 4, 5), while AIP displayed a homogeneous enhancement on PP/VP/DP $(P \leq 0.002)$. Massforming AIP frequently presented hyperintensity as compared with PDAC on PP/VP/DP $(P \leq 0.017)$.

\section{Mass enhancement contrast}

Table 3 summarizes the mass enhancement contrast results. Quantitative analysis showed that the mass enhancement contrast differed significantly between AIP and PDAC groups at all phases of DCE-MRI $(P \leq 0.003)$. In AIP, the lesion contrast which was the lowest at the unenhanced phase, increased gradually at the later phases. The contrast ultimately rose to $>1.0$ at PP $(9 / 17$, $0.53 \%)$, indicating AIP had high SI in contrast with NAP. In PDAC, the contrast was the lowest at the AP phase. It decreased from the unenhanced phase to the AP but progressively increased thereafter. However, the contrast remained $>1.0$ in most lesions $(14 / 17,82.4 \%)$ at the DP compared with PDAC. The VP was the maximum difference phase of the lesion contrast between AIP and PDAC. Sensitivity and specificity were $88.2 \%$ and $70.0 \%$, respectively, when the cutoff value for the Contrast ${ }_{\mathrm{VP}}$ value was 0.945 for differentiating AIP from PDAC. The AUROC curve was 0.859 .

\section{MRCP findings}

Regarding the MRCP features of AIP and PDAC, no significant differences were detected in the CBD abnormalities and penetrating duct sign. MPD stricture was more commonly detected in AIP than PDAC $(P=0.001)$. Furthermore, significant differences were also observed in skipped strictures of the MPD $(3 / 12,25 \%)$ and the side branch dilation $(1 / 12,8.3 \%)(P=0.019, P=0.004$, respectively). Only one AIP patient presented a dilated side branch of the pancreatic duct, whereas skipped strictures of the MPD were seen exclusively in AIP patients. However, the maximum diameter of the upstream MPD was not available for masses located in pancreatic tail, while that of the upstream MPD of 26 masses of PDAC [mean \pm standard deviation (SD), $5.1 \pm 2.4$; range: $1.0-$ $9.6 \mathrm{~mm}]$ was significantly larger than that of 9 masses of AIP (1.9 \pm 0.7 ; range: $1.2-3.5 \mathrm{~mm})$. When the cutoff value for the maximum diameter of the upstream MPD was set at $2.4 \mathrm{~mm}$ for distinguishing AIP from PDAC, a sensitivity of $88.9 \%$, specificity of $84.6 \%$, and an AUROC curve of 0.859 were obtained. The MRCP features are listed in Table 4.

\section{Quantitative analysis of DWI}

The results of the DWI findings are shown in Table 5 . The mean ADC value $\left(\times 10^{-3} \mathrm{~mm}^{2} / \mathrm{s}\right)$ of the masses was lower in mass-forming AIP than PDAC $(1.020 \pm 0.119$, $1.158 \pm 0.208$, respectively; $P=0.006$ ), while no statistical difference was revealed in NAP between AIP and PDAC at DWI $(1.246 \pm 0.185,1.233 \pm 0.166$, respectively; $P=0.818$ ). Also, there is no significant difference in the normalized ADC between AIP and PDAC $(P=0.048)$. When the cutoff value for the ADC was set at $1.099 \times 10^{-3} \mathrm{~mm}^{2} / \mathrm{s}$ in the ROC curve analysis for differentiation between the two groups, the sensitivity and specificity were $88.2 \%$ and $60.0 \%$. An AUROC curve of 0.733 was obtained. In addition, the mean diameter AIP was $34.5 \pm 24.3$ (range: $10.0-97.0$ ) $\mathrm{mm}$, which did not differ significantly from that of PDAC $(33.5 \pm 13.4 \mathrm{~mm}$, $15.7-70.1 \mathrm{~mm})(P=0.877)$. 

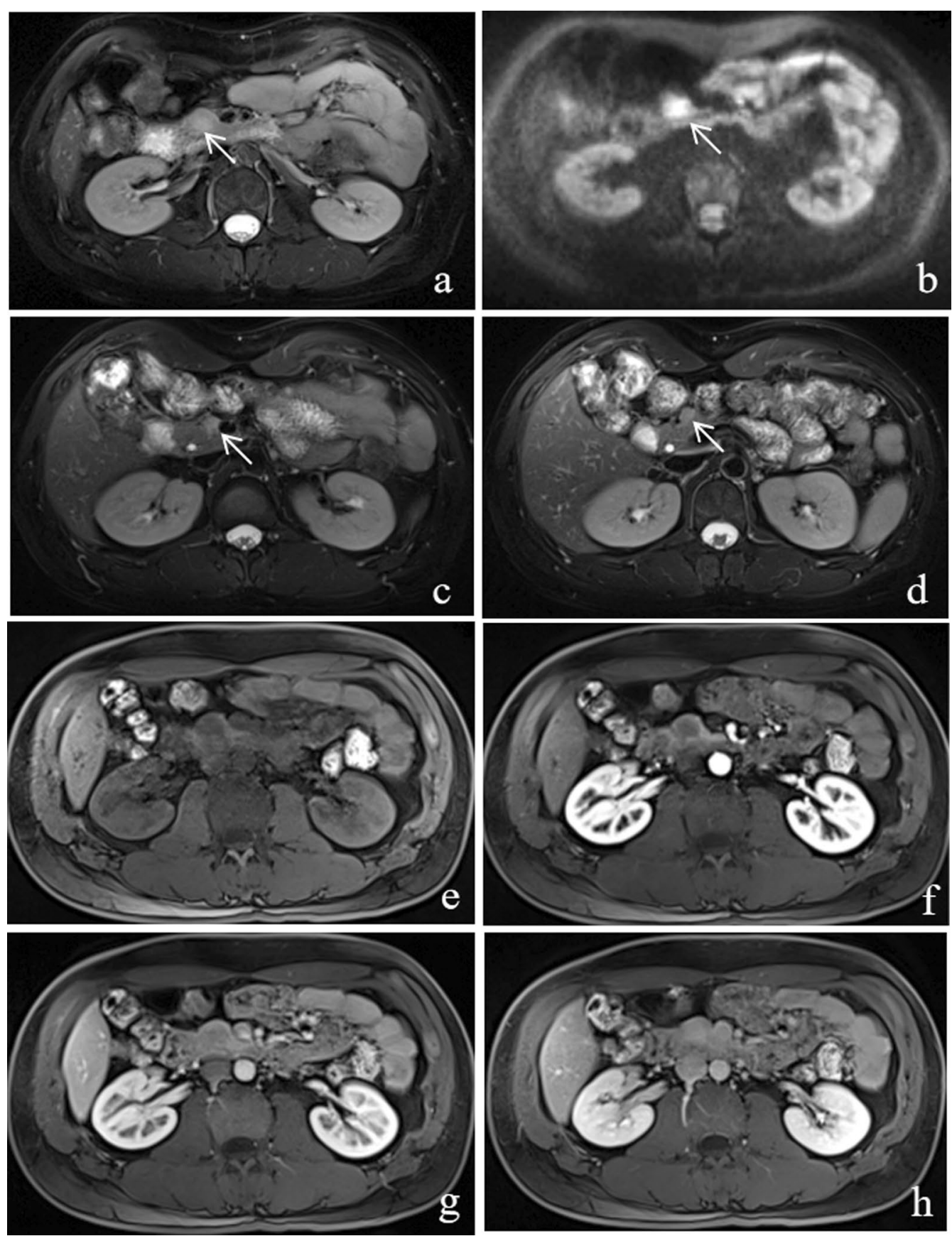

Fig. 2 A 30-year-old woman had mass-forming AIP with multiple masses in the pancreas. a T2WI, a round-shaped mass located in the pancreatic head (arrow) is observed as hyperintense. $\mathbf{b}$ On DWI $\left(b=800 \mathrm{~s} / \mathrm{mm}^{2}\right)$, the mass appears hyperintense compared to the pancreatic parenchyma (arrow). Another two small, round-shaped, hyperintensity masses are located in the pancreatic head (c) and neck (d) (arrows). e Unenhanced T1-weighted MR image exhibits a round hypointense nodule in the head of the pancreas. $\mathbf{f}$ On the AP image, the mass demonstrates a well-defined, obvious hypointensity. $\mathbf{g}$ On the PP image, the mass shows subtle hypo- or isointensity. $\mathbf{h}$ The DP image demonstrates the homogeneous hyperintensity of the mass

Table 6 shows the sensitivity and specificity for imaging findings. The venous phase homogeneous enhancement achieved a relatively high sensitivity of $70.6 \%$ and specificity of $93.3 \%$. The absence of internal cystic or necrotic portion had the highest sensitivity of $100.0 \%$, while the specificity was $33.3 \%$. The multiplicity, 

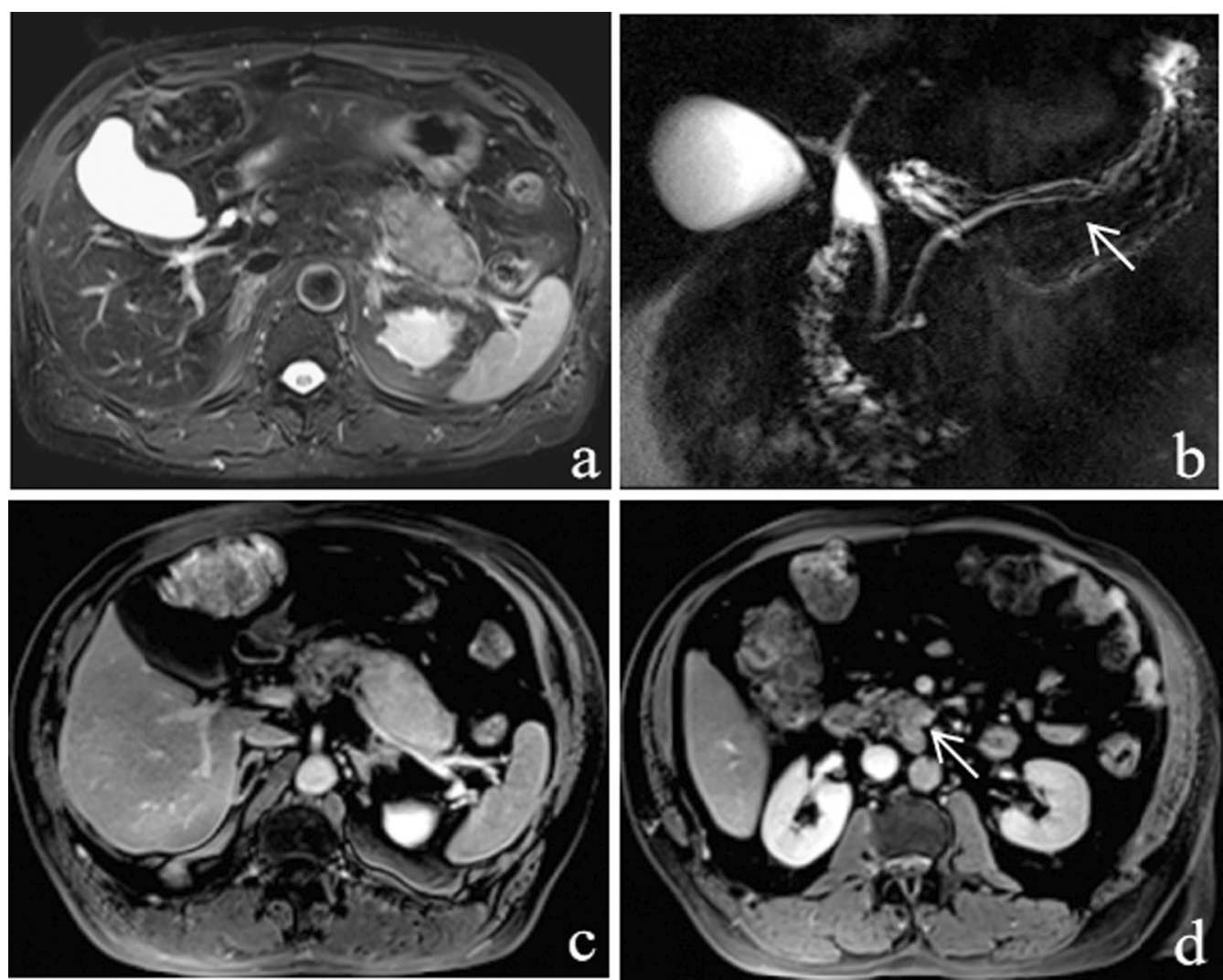

Fig. 3 A 63-year-old man had mass-forming AIP with two masses in the pancreas. a T2WI, an oval-shaped mass located in the pancreatic tail, is clearly observed as hyperintense with an indistinct margin. In addition, a hypointense rim is seen surrounding the pancreas. $\mathbf{b}$ MRCP image shows stenosis of the MPD of the pancreatic tail (penetrating duct sign) (arrow). c On the DP image, the mass shows hyperintensity with a peripancreatic enhancing rim (capsule-like rim enhancement). $\mathbf{d}$ A second area of lesion is observed in the pancreatic uncinate process (arrow). The area appears round and heterogeneously hyperintense

capsule-like rim enhancement and MPD skipped strictures had maximal specificity of $100.0 \%$, while the sensitivity was low.

\section{Discussion}

The imaging characteristics of mass-forming AIP using multimodel magnetic resonance imaging are critical for differentiation between mass-forming AIP and PDAC as misdiagnosis could lead to unnecessary invasive and surgical procedures [15-18].

The current study showed several statistically significant MR findings and quantitative indexes discriminating between the two conditions, and including multiplicity, irregular or geographical morphology, capsule-like rim enhancement, absence of internal cystic or a necrotic portion, homogeneous enhancement during the $\mathrm{PP} /$ VP/DP, skipped stricture or stricture of MPD, absence of side branch dilation, maximum upstream MPD diameter $<2.4 \mathrm{~mm}$, ADC value $<1.099 \times 10^{-3} \mathrm{~mm}^{2} / \mathrm{s}$,

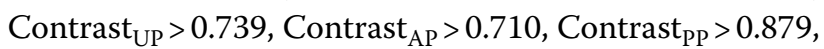
and Contrast ${ }_{\mathrm{VP}}$ or Contrast ${ }_{\mathrm{DP}}>0.949$; mass-forming AIP was more likely to exhibit these parameters together. Furthermore, AIP had the highest specificity in the imaging characteristics of multiplicity, capsule-like rim, and skipped stricture of MPD and hence, the possibility of PDAC could be excluded. Alternatively, internal cystic or a necrotic portion, side branch dilation, and heterogeneous enhancement during the PP/VP/DP suggested PDAC. Herein, 7 cases of mass-forming AIP were diagnosed by biopsy or even surgery. Based on the analysis of multimodel MRI findings, we had reason to conclude that our results were crucial for avoiding invasive or surgical procedures in mass-forming AIP that could be easily differentiated from PDAC.

One of representative imaging characteristics of AIP is the capsule-like rim that is used to distinguish AIP from PDAC. However, in recent studies, the sensitivity of the capsule-like rim sign with focal or massing AIP was low at $19.2-47.5 \%$ [19-22]. In our study, this feature was observed in 6/17 (35.3\%) masses with AIP; however, the major drawback was poor sensitivity. Similarly, multiplicity had high specificity (100.0\%) but poor sensitivity 

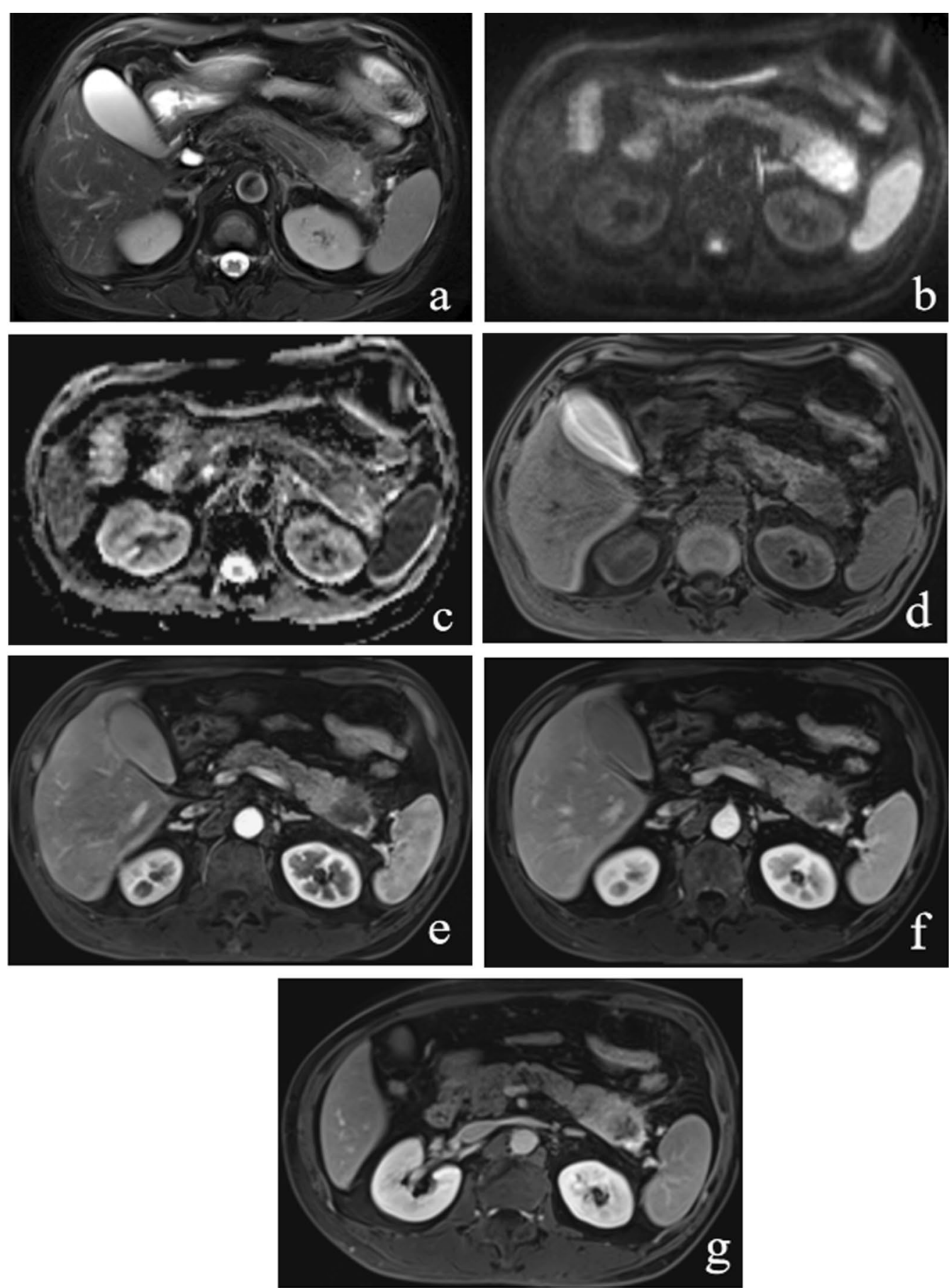

Fig. 4 A 62-year-old man had PDAC. a T2WI shows an oval-shaped mass located in the pancreatic tail that is visible as hyperintense with heterogeneity. $\mathbf{b}$ On DWI $\left(b=800 \mathrm{~s} / \mathrm{mm}^{2}\right)$ and ADC map (c), the pancreatic mass is clearly defined as hyperintense and hypointense, respectively. The pancreatic mass in the tail appears hypointense on both unenhanced (d) and (e) PP images. During the VP (f) and DP (g) imaging, the mass appears mild or obviously hyperintense, but the central area maintains hypointensity all the time

(33.3\%). Furthermore, the existence of multiple pancreatic masses can reflect the fact that the focal inflammatory masses can affect multiple areas of the pancreas.
Moreover, multimodel MRI findings include not only morphological and signal features of conventional MRI but also DCE-MRI and DWI, which has an outstanding 

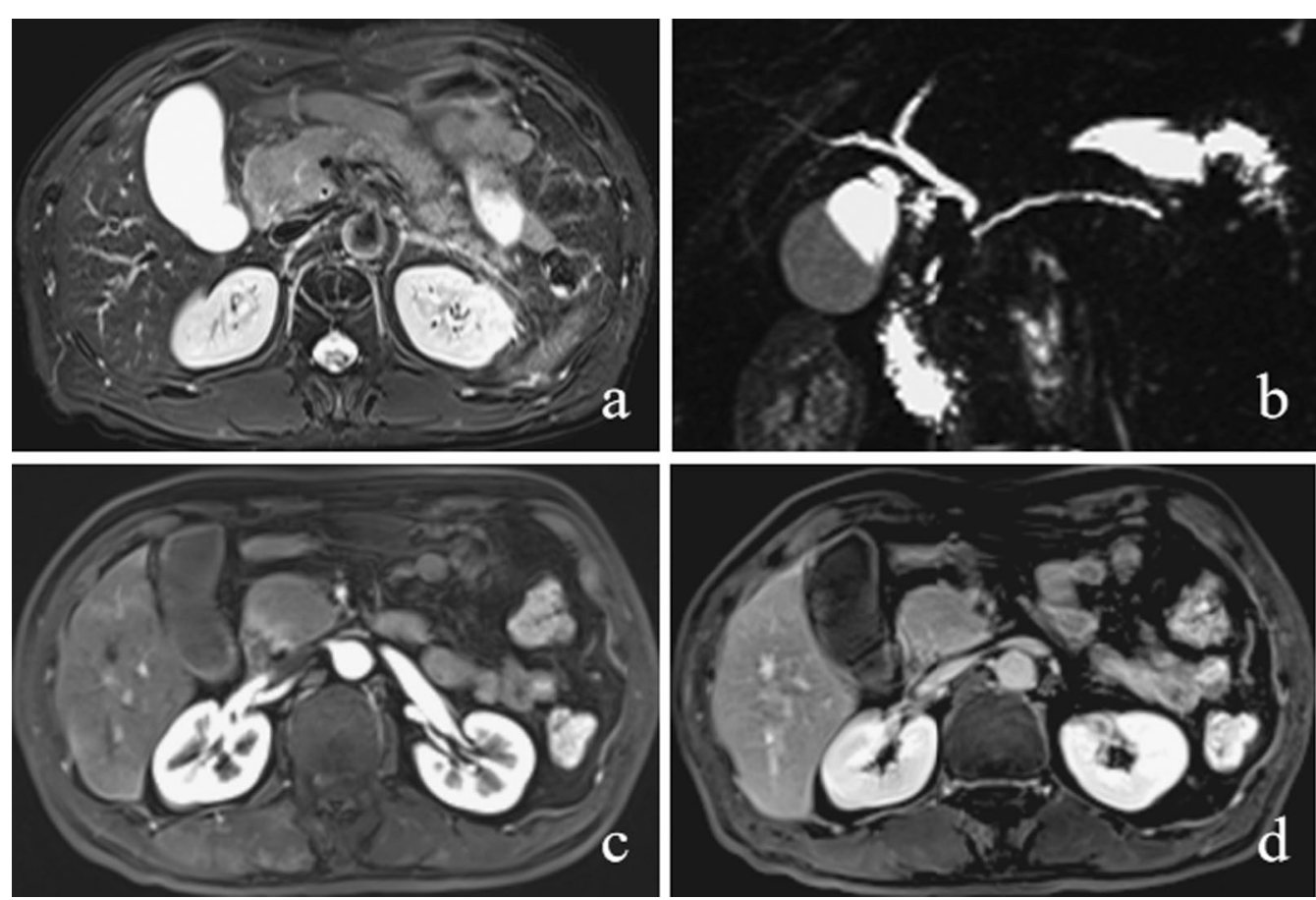

Fig. 5 A 57-year-old man had PDAC. a T2WI, the round pancreatic head mass shows hyperintensity with homogeneity. b MRCP image shows the cut-off of the CBD and MPD. On the PP image (c) shows the mass with hypointensity. $\mathbf{d}$ On the DP image, the mass shows homogeneous enhancement

Table 3 Quantitative analysis of the mass enhancement contrast

\begin{tabular}{|c|c|c|c|c|c|}
\hline & AIP $(N=17)$ & $\operatorname{PDAC}(N=30)$ & $P$ value & ACROC* & $P$ value \\
\hline Contrast $_{\text {UP, }}$, mean (range, SD) & $0.79(0.55-1.06,0.16)$ & $0.66(0.44-0.98,0.11)$ & 0.003 & $0.735(0.579-0.892)$ & 0.172 \\
\hline Contrast $_{\mathrm{AP}}$, mean (range, SD) & $0.85(0.45-1.18,0.21)$ & $0.55(0.22-1.16,0.19)$ & $<0.001$ & $0.851(0.734-0.968)$ & 0.885 \\
\hline Contrast $_{p p}$, mean (range, SD) & $1.10(0.73-1.63,0.26)$ & $0.70(0.21-1.38,0.28)$ & $<0.001$ & $0.851(0.745-0.957)$ & 0.733 \\
\hline Contrast $_{\mathrm{VP},}$ mean (range, SD) & $1.28(0.83-1.92,0.34)$ & $0.79(0.24-1.48,0.30)$ & $<0.001$ & $0.859(0.755-0.963)$ & \\
\hline Contrast $_{D P}$, mean (range, SD) & $1.30(0.83-1.79,0.28)$ & $1.00(0.30-1.53,0.33)$ & 0.003 & $0.749(0.607-0.891)$ & 0.009 \\
\hline
\end{tabular}

$U P$ unenhanced phase, $A P$ arterial phase, $P P$ pancreatic phase, $V P$ portal venous phase, $D P$ delayed phase

*AUROC for differentiating between AIP and PDAC (95\% Cl)

\# Compared to AUROC of Contrast $\mathrm{VP}$

ability to detect multiple masses, especially a subtle lesion. Typically, the pancreatic mass observed in AIP showed an irregular or geographical morphology, unlike the oval or round shape observed in PDAC.

Our study detected that quantitative analysis of the mass enhancement contrast using DCE-MRI could be valuable for differentiating between the two conditions. Furthermore, the quantitative analysis results suggested that Contrast $_{\mathrm{VP}}$ can be used as a vital index in the differentiation between AIP and PDAC in view of its excellent diagnostic performance when compared with other phase indexes. Kwon et al. [22] reported that Contrast ${ }_{\mathrm{AP}}$ had excellent diagnostic performance for differentiating between the two diseases which was in disaggrement with our study. We assume that this discrepancy may arise from difference in MRI parameters or in criteria for inclusion of the study population. We analyzed all lesions, including small ones. Interestingly, we observed the homogenous enhancement on PP which was significantly more frequent in mass-forming AIP than in PDAC. These results suggested that AIP with multiple masses showed early homogeneous enhancement, which could be attributed to the small size of some lesions and focal inflammation. Previous studies had reported the key imaging feature of delayed homogeneous enhancement in mass-forming AIP [9, 20-25], which was consistent 
Table 4 MRCP findings between AIP and PDAC

\begin{tabular}{|c|c|c|c|c|c|}
\hline MRCP findings & $\operatorname{AIP}(N=12)$ & $k$ & $\operatorname{PDAC}(N=30)$ & $k$ & $P$ value \\
\hline CBD & & 0.867 & & 0.795 & 0.570 \\
\hline Normal & 6 & & 13 & & \\
\hline Stricture & 4 & & 7 & & \\
\hline Complete obstruction & 2 & & 10 & & \\
\hline CBD skipped strictures & 1 & 1 & 0 & 1 & 0.286 \\
\hline MPD & & 0.871 & & 0.927 & 0.001 \\
\hline Normal & 0 & & 4 & & \\
\hline Stricture & 6 & & 1 & & \\
\hline Complete obstruction & 3 & & 21 & & \\
\hline Not visible & 3 & & 4 & & \\
\hline MPD skipped strictures & 3 & 0.750 & 0 & 1 & 0.019 \\
\hline Side branch dilation & 1 & 1 & 17 & 0.733 & 0.004 \\
\hline Penetrating duct sign & 2 & 1 & 0 & 1 & 0.077 \\
\hline $\begin{array}{l}\text { Maximum upstream MPD diameter } \\
(\mathrm{mm})^{*}\end{array}$ & $1.9 \pm 0.7(1.2-3.5)$ & - & $5.1 \pm 2.4(1.0-9.6)$ & - & $<0.001$ \\
\hline
\end{tabular}

Numbers are the number of patients

*Masses located on the edge of the pancreatic tail were excluded in 4 AIP patients. Data are means \pm standard deviation with the ranges in parentheses

with the current findings. Pathologically, both AIP and PDAC presented abundant fibrous stroma, which usually resulted in delayed enhancement. However, heterogeneous enhancement of the lesions of PDAC has frequently been observed on DCE-MRI because of necrosis, cyst degeneration, or hemorrhage [26-28].

In this study, the mean ADC value of masses is lower in AIP than in PDAC, while no statistical difference was observed in the SI on DWI, NAP ADC value, and the normalized ADC between the two conditions. When the ADC cutoff value in distinguishing mass-forming AIP from PDAC was $1.099 \times 10^{-3} \mathrm{~mm}^{2} / \mathrm{s}$, a sensitivity of $88.2 \%$ and specificity of $60.0 \%$ was obtained. Several studies reported different ADC optimal cutoff values, ranging from $0.88-1.26 \times 10^{-3} \mathrm{~mm}^{2} / \mathrm{s}$, so as to differentiate mass-forming AIP from PDAC. Our cutoff value of ADC is in the range reported in previous studies, although our specificity was the lowest $[1,9,20,29]$. However, the reason why mass-forming AIP had a lower mean ADC value

Table 5 Results of ADC value and normalized ADC

\begin{tabular}{lccl}
\hline & AIP & PDAC & P value \\
\hline Diameter $(\mathrm{mm})$ & $34.5 \pm 24.3$ & $33.5 \pm 13.4$ & 0.877 \\
Mass ADC value $\left(\times 10^{-3} \mathrm{~mm}^{2} / \mathrm{s}\right)$ & $1.020 \pm 0.119$ & $1.158 \pm 0.208$ & 0.006 \\
NAP ADC value $\left(\times 10^{-3} \mathrm{~mm}^{2} / \mathrm{s}\right)$ & $1.246 \pm 0.185$ & $1.233 \pm 0.166$ & 0.818 \\
Normalized ADC* & $0.838 \pm 0.130$ & $0.953 \pm 0.205$ & 0.048 \\
\hline
\end{tabular}

Data are means \pm standard deviation in parentheses; NAP, non-mass adjacent pancreatic parenchyma; Normalized ADC, pancreatic mass to non-mass adjacent pancreatic parenchymal ADC ratio. Normalized $A D C^{*}$ was available in 16 lesions of AIP and in 30 lesions of PDAC than PDAC is yet to be elucidated. We presume that the lymphoplasmacytic infiltration and fibrous tissue in AIP is more abundant than that of PDAC, which includes diffuse infiltration of cancer cells and abundant fibrosis component [29]. Increased cellularity, chronic fibroinflammatory processes, and pancreatic tissue edematous changes may play critical roles in achieving the lower ADC values in mass-forming AIP than in PDAC [29]. In addition, a common internal cystic or necrotic portion in PDAC was responsible for obtaining high ADC values $[1,29]$. These factors may contribute to the difference in ADC value observed in this study between the two groups. Together, the current and previous results $[1,9$, 20] have demonstrated that DWI is vital for the detection of mass-forming AIP especially multifocal or small lesions as well as the differentiation of AIP from PDAC.

In this study, only AIP showed skipped strictures of MPD, which might be an indicator of mass-forming AIP that presented skipped MPD narrowing, less complete obstruction MPD, less dilated upstream MPD, and less side branch dilation on MRCP. These results were consistent with those reported previously $[8,19,20]$. However, there were no statistically significant difference between mass-forming AIP and PDAC regarding CBD abnormalities or penetrating duct sign. As reported in the literature, a prevalence of skipped strictures of MPD was observed to be developed along with the whole extension of the MPD in the patients with multiple stenoses even if the lesions involved were segmental [19]. This finding of skipped strictures of MPD may reflect the extension of inflammatory infiltration which can lead to focal 
Table 6 Sensitivity and specificity of the significant MR findings in the diagnosis of mass-forming AIP

\begin{tabular}{|c|c|c|}
\hline & Sensitivity (\%) & Specificity (\%) \\
\hline Multiplicity & $33.3(4 / 12)$ & $100(28 / 28)$ \\
\hline Irregular or geographical shape & $52.9(9 / 17)$ & $90(27 / 30)$ \\
\hline Capsule-like rim enhancement & $35.3(6 / 17)$ & $100(30 / 30)$ \\
\hline Absence of internal cystic or necrotic portion & $100(17 / 17)$ & $33.3(10 / 30)$ \\
\hline Absence of side branch dilation & $91.7(11 / 12)$ & $56.7(17 / 30)$ \\
\hline Pancreatic phase homogeneous enhancement & $47.1(8 / 17)$ & $93.3(28 / 30)$ \\
\hline Venous phase homogeneous enhancement & $70.6(12 / 17)$ & $93.3(28 / 30)$ \\
\hline Delayed phase homogeneous enhancement & $76.5(13 / 17)$ & $93.3(28 / 30)$ \\
\hline MPD stricture & $50.0(6 / 12)$ & $96.7(29 / 30)$ \\
\hline MPD skipped strictures & $25.0(3 / 12)$ & $100(30 / 30)$ \\
\hline Maximum upstream MPD diameter $<2.4 \mathrm{~mm}$ & $88.9(8 / 9)$ & $84.6(22 / 26)$ \\
\hline ADC value $\left.<1.099 \times 10^{-3} \mathrm{~mm}^{2} / \mathrm{s}\right)$ & $88.2(15 / 17)$ & $60.0(18 / 30)$ \\
\hline Contrast $_{\text {UP }}>0.737$ & $64.7(11 / 17)$ & $83.3(25 / 30)$ \\
\hline Contrast $_{\mathrm{AP}}>0.690$ & $82.4(14 / 17)$ & $83.3(25 / 30)$ \\
\hline Contrast $_{p p}>0.862$ & $82.4(14 / 17)$ & $76.7(23 / 30)$ \\
\hline Contrast $_{\mathrm{VP}}>0.945$ & $88.2(15 / 17)$ & $70.0(21 / 30)$ \\
\hline Contrast $_{D P}>0.942$ & $88.2(15 / 17)$ & $50.0(15 / 30)$ \\
\hline
\end{tabular}

The data in parentheses represent the number of patients or masses

enlargement of the pancreas. The inflammatory compression of AIP leads to a mild dilation of the MPD upstream dilatation (mean $=2.4 \mathrm{~mm}$ in our study) unlike PDAC, in which $\mathrm{CBD}$ or pancreatic duct abnormalities are induced by cancer cell infiltrating the ductal system; consequently, MPD cutoff or marked dilation of the upstream MPD or side branch dilation were also observed. Previous study revealed that administration of secretin in AIP could reduce number and length of MPD stenoses [20, 30, 31]. Secretin administration enhances the performance of MRCP, especially the evidence of penetrating duct sign. Based on these results, adding MRCP to conventional MRI and DCE-MRI would be significant for diagnosing AIP as it provides additional information of CBD and MPD.

Nevertheless, this study has some limitations. First, the possibility of selection bias due to the retrospective study design should be considered. Second, the number of patients with mass-forming AIP was limited, and only 12 patients were available for MRI examination. Third, the lesion size was small in some cases of mass-forming AIP, which might have biased the determination of signal intensity qualitatively and quantitatively.

\section{Conclusions}

We identified characteristic MR findings that can differentiate mass-forming from PDAC. Multimodel MRI, including unenhanced MRI, DCE-MRI with DWI and $\mathrm{MRCP}$ can provide qualitative and quantitative information about mass-forming AIP characterization. Multimodel MRI can be helpful for the appropriate diagnosis of mass-forming AIP.

\section{Abbreviations}

MRI: Magnetic resonance imaging; DCE-MRI: Dynamic contrast-enhanced MRI; MRCP: MR-cholangiopancreatography; DWI: Diffusion-weighted imaging; AIP: Autoimmune pancreatitis; PDAC: Pancreatic ductal adenocarcinoma.

\section{Acknowledgements}

Not applicable.

\section{Authors' contributions}

GWL contributed to the study conception and designed the study. Data collection was performed by HHJ, GWL and WJH. HHJ and JLL performed the statistical analysis. HHJ wrote the main manuscript text. WJH prepared Figs. 1, 2, 3 and 4. All the authors contributed to the data analysis and interpretation.

All authors read and approved the final manuscript.

\section{Funding}

This study was not funded by any organization.

\section{Availability of data and materials}

The datasets analyzed in this study are available from the corresponding author on request.

\section{Declarations}

\section{Ethics approval and consent to participate}

The Institutional Review Board of Huadong Hospital approved this retrospective study and waived the requirement for written informed consent due to its retrospective nature.

Consent for publication

Not applicable. 


\section{Competing interests}

The authors declare that they have no competing interests.

\section{Author details}

'Department of Radiology, Huadong Hospital Affiliated to Fudan University, 221 West Yanan Road, Shanghai 200040, China. ${ }^{2}$ Department of General Surgery, Center of Pancreaticobiliary Disease, Huadong Hospital Affiliated to Fudan University, Shanghai, China.

Received: 2 July 2021 Accepted: 28 September 2021

Published online: 15 October 2021

\section{References}

1. Choi SY, Kim SH, Kang TW, Song KD, Park HJ, Choi YH. Differentiating mass-forming autoimmune pancreatitis from pancreatic ductal adenocarcinoma on the basis of contrast-enhanced MRI and DWI findings. AJR Am J Roentgenol. 2016;206(2):291-300.

2. Sureka B, Rastogi A. Autoimmune pancreatitis. Pol J Radiol. 2017;82:233-9.

3. Manfredi R, Frulloni L, Mantovani W, Bonatti M, Graziani R, Pozzi MR. Autoimmune pancreatitis: pancreatic and extrapancreatic MR imaging-MR cholangiopancreatography findings at diagnosis, after steroid therapy, and at recurrence. Radiology. 2011:260(2):428-36.

4. O'Reilly DA, Malde DJ, Duncan T, Rao M, Filobbos R. Review of the diagnosis, classification and management of autoimmune pancreatitis. World J Gastrointest Pathophysiol. 2014;5(2):71-81.

5. Negrelli R, Boninsegna E, Avesani G, Zamboni GA, Brozzi L, Frulloni L, et al. Type 1 and type 2 autoimmune pancreatitis: distinctive clinical and pathological features, but are there any differences at magnetic resonance? Experience from a referral center. Pancreas. 2018:47(9):1115-22.

6. Hart PA, Zen Y, Chari ST. Recent advances in autoimmune pancreatitis. Gastroenterology. 2015;149(1):39-51.

7. Hafezi-Nejad N, Singh VK, Fung C, Takahashi N, Zaheer A. MR imaging of autoimmune pancreatitis. Magn Reson Imaging Clin N Am. 2018;26(3):463-78.

8. Lopes Vendrami C, Shin JS, Hammond NA, Kothari K, Mittal PK, Miller FH Differentiation of focal autoimmune pancreatitis from pancreatic ductal adenocarcinoma. Abdom Radiol. 2020;45(5):1371-86.

9. Muhi A, Ichikawa T, Motosugi U, Sou H, Sano K, Tsukamoto T, et al. Mass-forming autoimmune pancreatitis and pancreatic carcinoma: differential diagnosis on the basis of computed tomography and magnetic resonance cholangiopancreatography, and diffusion-weighted imaging findings. J Magn Reson Imaging JMRI. 2012;35(4):827-36.

10. Al-Hawary MM, Kaza RK, Azar SF, Ruma JA, Francis IR. Mimics of pancreatic ductal adenocarcinoma. Cancer Imaging Off Publ Int Cancer Imaging Soc. 2013;13(3):342-9.

11. Sugiyama Y, Fujinaga Y, Kadoya M, Ueda K, Kurozumi M, Hamano $H$, et al. Characteristic magnetic resonance features of focal autoimmune pancreatitis useful for differentiation from pancreatic cancer. Jpn J Radiol. 2012;30(4):296-309.

12. Maruyama M, Watanabe T, Kanai K, Oguchi T, Muraki T, Hamano H, et al. International consensus diagnostic criteria for autoimmune pancreatitis and its Japanese amendment have improved diagnostic ability over existing criteria. Gastroenterol Res Pract. 2013;2013:456965.

13. Madhani $\mathrm{K}$, Farrell JJ. Autoimmune pancreatitis: an update on diagnosis and management. Gastroenterol Clin N Am. 2016;45(1):29-43.

14. Okazaki K, Uchida K. Current perspectives on autoimmune pancreatitis and lgG4-related disease. Proc Jpn Acad Ser B Phys Biol Sci. 2018;94(10):412-27.
15. Agrawal S, Daruwala C, Khurana J. Distinguishing autoimmune pancreatitis from pancreaticobiliary cancers: current strategy. Ann Surg. 2012;255(2):248-58

16. Kim JH, Kim MH, Byun JH, Lee SS, Lee SJ, Park SH, et al. Diagnostic strategy for differentiating autoimmune pancreatitis from pancreatic cancer: is an endoscopic retrograde pancreatography essential? Pancreas. 2012;41(4):639-47.

17. Hedfi M, Charfi M, Nejib FZ, Benlahouel S, Debaibi M, Ben Azzouz S, et al. Focal mass-forming autoimmune pancreatitis mimicking pancreatic cancer: which strategy? Tunis Med. 2019;97(5):731-5.

18. Wolske KM, Ponnatapura J, Kolokythas O, Burke LMB, Tappouni $R$, Lalwani N. Chronic pancreatitis or pancreatic tumor? A problem-solving approach. Radiographics. 2019;39(7):1965-82.

19. Negrelli R, Manfredi R, Pedrinolla B, Boninsegna E, Ventriglia A, Mehrabi $S$, et al. Pancreatic duct abnormalities in focal autoimmune pancreatitis: MR/MRCP imaging findings. Eur Radiol. 2015;25(2):359-67.

20. Hur BY, Lee JM, Lee JE, Park JY, Kim SJ, Joo I, et al. Magnetic resonance imaging findings of the mass-forming type of autoimmune pancreatitis: comparison with pancreatic adenocarcinoma. J Magn Reson Imaging JMRI. 2012;36(1):188-97.

21. Lee S, Kim JH. Comparison of diagnostic performance between CT and MRI in differentiating non-diffuse-type autoimmune pancreatitis from pancreatic ductal adenocarcinoma. Eur Radiol. 2018;28(12):5267-74.

22. Kwon JH, Kim JH. Differentiating focal autoimmune pancreatitis and pancreatic ductal adenocarcinoma: contrast-enhanced MRI with special emphasis on the arterial phase. Eur Radiol. 2019;29(11):5763-71.

23. Rehnitz C, Klauss M, Singer R, Ehehalt R, Werner J, Büchler MW, et al. Morphologic patterns of autoimmune pancreatitis in CT and MRI. Pancreatol Off J Int Assoc Pancreatol IAP. 2011;11(2):240-51.

24. Nagpal SJS, Sharma A, Chari ST. Autoimmune pancreatitis. Am J Gastroenterol. 2018;113(9):1301.

25. Tang CSW, Sivarasan N, Griffin N. Abdominal manifestations of IgG4related disease: a pictorial review. Insights Imaging. 2018;9(4):437-48.

26. Balci NC, Semelka RC. Radiologic diagnosis and staging of pancreatic ductal adenocarcinoma. Eur J Radiol. 2001;38(2):105-12.

27. Kosmahl M, Pauser U, Anlauf M, Klöppel G. Pancreatic ductal adenocarcinomas with cystic features: neither rare nor uniform. Mod Pathol Off J U S Can Acad Pathol. 2005;18(9):1157-64.

28. D'Onofrio M, De Robertis R, Capelli P, Tinazzi Martini P, Crosara S, Gobbo $\mathrm{S}$, et al. Uncommon presentations of common pancreatic neoplasms: a pictorial essay. Abdom Imaging. 2015;40(6):1629-44.

29. Kamisawa T, Takuma K, Anjiki H, Egawa N, Hata T, Kurata M, et al. Differentiation of autoimmune pancreatitis from pancreatic cancer by diffusionweighted MRI. Am J Gastroenterol. 2010;105(8):1870-5.

30. Carbognin G, Girardi V, Biasiutti C, Camera L, Manfredi R, Frulloni L, et al. Autoimmune pancreatitis: imaging findings on contrast-enhanced MR, MRCP and dynamic secretin-enhanced MRCP. Radiol Med (Torino). 2009;114(8):1214-31.

31. Boninsegna E, Manfredi R, Negrelli R, Avesani G, Mehrabi S, Pozzi MR. Pancreatic duct stenosis: differential diagnosis between malignant and benign conditions at secretin-enhanced MRCP. Clin Imaging. 2017:41:137-43.

\section{Publisher's Note}

Springer Nature remains neutral with regard to jurisdictional claims in published maps and institutional affiliations. 